\title{
Cardiac arrest during a sub-tenon block: a sign of acute lidocaine and bupivacaine toxicity
}

\author{
Luke Masha $^{1 *}$ and Eric Awtry ${ }^{2}$ \\ ${ }^{1}$ Internal Medicine, Boston University, Boston, MA, USA \\ ${ }^{2}$ Section of Cardiology, Boston University, Boston, MA, USA
}

\begin{abstract}
The neurologic and cardiac toxicities of local anesthetics lidocaine and bupivacaine have long been documented and can be potentiated by inadvertent systemic administration, pre-existing cardiac disease, shock states, and renal failure. We report a peculiar case of acute lidocaine and bupivacaine toxicity during orbital surgery in a woman with stable chronic systolic heart failure due to unexpected, rapid systemic absorption through a carotid-cavernous fistula. This case was marked by persistent bi-hemispheric coma, cardiac arrest, and ultimately death from repeated bradycardic pulseless electrical activity despite the presence of a functional cardiac pacemaker. Lidocaine and Bupivacaine toxicities are discussed as well as complications of orbital surgery which may mimic them.
\end{abstract}

\section{Introduction}

Severe neurologic and cardiac adverse effects of the local anesthetics lidocaine and bupivacaine are rare. However, when there is inadvertently rapid systemic exposure, serious neurologic and cardiovascular toxicity may occur.

\section{Case report}

A 57 year old woman was referred for elective pars plana vitrectomy of the left eye as management of a retinal detachment. She had a medical history significant for NYHA Class II heart failure from coronary artery disease, with an estimated left ventricular ejection fraction of $15-20 \%$. She was one year status post placement of a St Jude Fortify VR single chamber ICD with no cardiac events within that interval. She weighed 50.7 kilograms and took $50 \mathrm{mg}$ of metoprolol succinate twice daily, $40 \mathrm{mg}$ of furosemide daily, and $25 \mathrm{mg}$ of spironolactone daily. She reported ambulation of four blocks without development of dyspnea. Pre-operative labs demonstrated a normal complete blood count and chemistry panel. Her baseline ECG revealed normal sinus rhythm, an old antero-septal infarct pattern, and nonspecific anterolateral ST segment abnormalities. She took one dose of metoprolol on the morning of presentation.

In the operating room, she received $50 \mathrm{mcg}$ of remifentanil without event. Uncomplicated blunt dissection for a sub-tenon block [1] was performed. Instillation of $3 \mathrm{ml}$ of a 1:1 mixture of $2 \%$ lidocaine and $0.75 \%$ bupivacaine into the posterior subtenon space was performed. Total administered lidocaine and bupivacaine doses respectively were $30 \mathrm{mg}$ and $11.25 \mathrm{mg}$. The conjunctival incision was closed with a suture. At this point, she was noted to be hypotensive, bradycardic, apneic, and unresponsive to stimulation. Cardiopulmonary resuscitation (CPR) and prompt intubation without sedation were initiated. Her pupils and optic discs were found to be intact, without evidence of increased intracranial or intraorbital pressure. Telemetry revealed demand pacing at 40 beats per minute (bpm). Due to concerns for triggering an oculocardiac reflex, she received glycopyrrolate and atropine without response. After the administration of epinephrine, she achieved return of spontaneous circulation 2 minutes after initiation of CPR. Following resuscitation, her examination was consistent with bi-hemispheric coma. An emergent cranial computed tomography angiogram (CTA) revealed no acute vessel occlusion or intracranial hemorrhage. It revealed dilation of the superior ophthalmic vein with associated prominence of its tributaries, asymmetric early enhancement of the left cavernous sinus, left sigmoid vein and left internal jugular vein in addition to increased prominence of the veins in the left pterygoid venous plexus and left retromandibular vein (Figures 1-5). These findings were concerning for a carotid-cavernous fistula vs a dural arteriovenous fistula.

While undergoing the CTA, she again developed profound bradycardia and hypotension requiring CPR. She received several doses of atropine without response but did respond to a dopamine infusion. ICD interrogation appeared normal, with acceptable capture and pacing voltages. The demand pacing lower limit threshold was increased to $60 \mathrm{bpm}$ at this time. The patient's hospital course over a twenty hour period was marked by repeated episodes of pulselessness associated with severe bradycardia requiring CPR, despite escalation of vasopressors and demand pacing lower limit thresholds. Interspersed ECGs showed sinus tachycardia with a persistently prolonged PR. Rarely deep antero-lateral ST segment depressions could be seen. Chemistries were unremarkable outside of a rapidly rising creatinine. Her echocardiogram showed an EF of $10-15 \%$, but was otherwise unchanged. Troponin I measurements peaked at $18 \mathrm{ng} / \mathrm{ml}$. Left

Correspondence to: Luke Masha, Internal Medicine, Boston University, 72 East Concord Street, Evans 124, Boston, MA 02118, USA, Tel: 617-638-6500; Fax: 617-638-6501; E-mail: Luke.Masha@bmc.org

Key words: cardiac arrest, Lidocaine, Bupivacaine, Cardiotoxicity, Anesthetics local, Orbital surgery

Received: March 08, 2015; Accepted: April 05, 2015; Published: April 07, 2015 


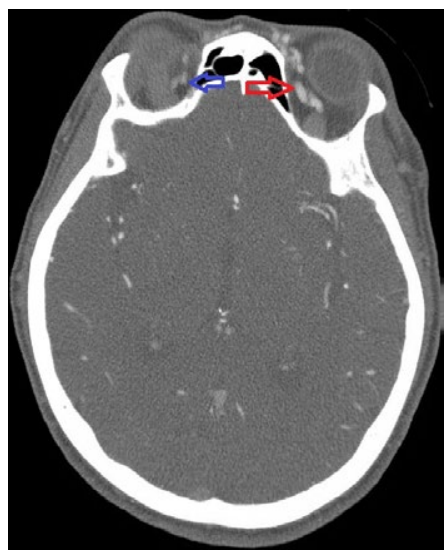

Figure 1. Asymmetric enhancement of the left ophthalmic vein. Image is captured in the arterial phase.

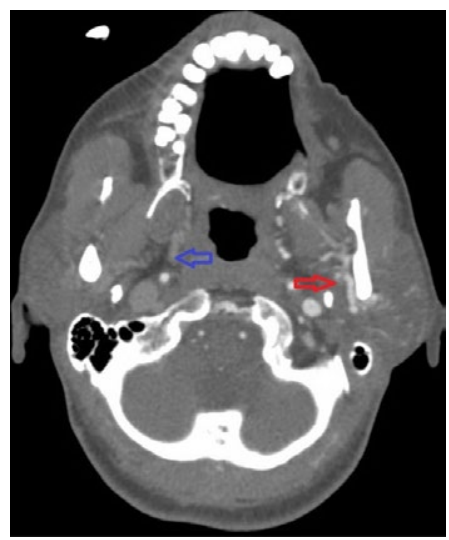

Figure 2. Asymmetric enhancement of the left pterygoid plexus. Image is captured in the arterial phase.

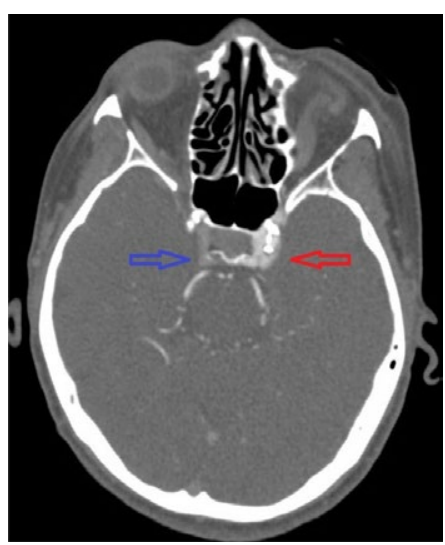

Figure 3. Asymmetric enhancement of the left cavernous sinus. Image is captured in the arterial phase.

heart catherization was recommended. However, her neurologic status remained consistent with a profound bi-hemispheric coma and ultimately due to a poor overall prognosis, care was ultimately withdrawn by her next of kin.

\section{Discussion}

Orbital surgery carries well described complications worth

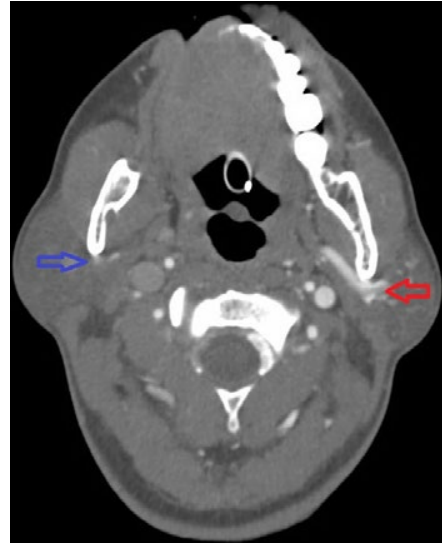

Figure 4. Asymmetric enhancement of the left retromandibular vein. Image is captured in the arterial phase.

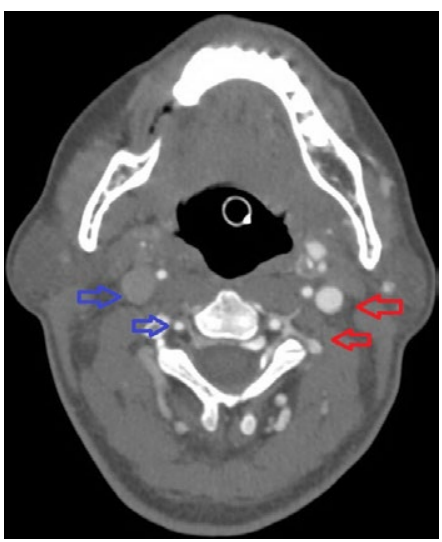

Figure 5. Asymmetric enhancement of the left internal jugular vein and left vertebral venous plexus. Image is captured in the arterial phase.

discussion as they may initially mimic this presentation: the oculocardiac reflex and the diving reflex [2,3]. The oculocardiac reflex may be triggered by any orbital sensory stimulation. This leads to activation of the ophthalmic division of the trigeminal nerve, which has afferent synapses with the visceral motor nucleus of the vagus nerve. In susceptible individuals, powerful vagal activation occurs and may lead to profound bradycardia. The pathophysiology for the more potent diving reflex is similar; however the latter is associated with cool stimuli and cold-induced peripheral vasoconstriction. These reflexes usually resolve with removal of the sensory stimulus and can be blocked entirely with atropine and glycopyrrolate. Moreover there is evidence that lidocaine administration attenuates the afferent limb of these reflexes and reduces their incidence [4]. This knowledge, when considered with her normal eye exam, the failure of response to atropine and glycopyrrolate, and the occurrence of multiple events after stimulus removal argues against neurocardiogenic reflexes as being the cause of her repeated events.

The patient's marked troponin elevation suggested the possibility of an acute myocardial infarction (MI). Acute marked bradycardia is an uncommon, but possible presentation of an acute MI. However an acute MI is unlikely to represent her initial event in the operating room based on numerous clinical cues. An ischemic event significant enough to cause immediate cardiovascular collapse would likely be associated with significant ECG findings. Such changes were absent and her echocardiogram was also grossly unchanged. In addition, there were 
no clinical exam findings of acute heart failure secondary to possible large acute MI. Furthermore, her neurologic exam of a persistent bihemispheric coma would be unexpected in an acute MI associated with rapid and successful resuscitation. Her significant troponin elevation rather was a marker of repeated demand ischemia in the setting of several episodes of hypotension and inotrope induced tachycardia in a patient with coronary artery disease.

Her initial bradycardic event most likely represented acute cardiac and CNS toxicity from lidocaine and bupivacaine administration. The temporal association of her initial bradycardia with injection of these agents is strongly suggestive, especially in light of possible systemic absorption via an arteriovenous malformation such as a carotid-cavernous fistula. In addition, PR prolongation in the setting of tachycardia is unexpected and is an ECG finding commonly associated with local anesthetic cardiotoxicity. If larger doses of lidocaine or bupivacaine were involved, almost certainly would such toxicity be the diagnosis. However lidocaine toxicity leading to profound bradycardia, asystole and circulatory collapse has been well described following 30 mg-50 mg IV boluses [5-7] as such doses, depending on a number of clinical factors, may inadvertently result in toxic serum levels $(>10$ $\mathrm{mcg} / \mathrm{ml}$ ). The cardiotoxic dose of bupivacaine in humans is unknown. However bupivacaine is estimated to be 20x more cardiotoxic than lidocaine, and is associated with difficult and prolonged resuscitations, refractory arrhythmias, and a high mortality. Case reports of sudden cardiac arrest from bupivacaine have involved systemic exposure to doses as little as $6 \mathrm{ml}$ of a $0.25 \%$ solution in children [8] and $15-20 \mathrm{ml}$ of a $0.5 \%$ solution in adults [9]. Furthermore, administration of as little as $2 \mathrm{ml}$ of a $0.75 \%$ may result in ventricular tachycardia in healthy patients [9]. The half-life of lidocaine after direct systemic administration is approximately $2.5-4$ hours and that of bupivacaine is 3.5 hours. With time, these drugs should distribute from the serum into the tissue beds as well as be metabolized by the liver and kidneys. However, it should be noted that the drug half-lives are prolonged in CHF and shock states, when cardiac output is shunted away from the liver and kidneys and directed preferentially to the heart and brain. In terms of lidocaine and bupivacaine, this unfortunately results in their administration into a smaller initial volume of distribution, decreased hepatic metabolism and renal clearance, higher than expected plasma concentrations, and preferential and progressive uptake by the heart and brain.

The persistent bi-hemispheric coma was unexpected given a relatively short resuscitation time and suggests CNS toxicity from the administered anesthetics. Such toxicity initially involves an excitatory phase marked by myoclonus and seizures. As CNS drug levels increase, complete depression of conscious activity occurs as neural tissue sodium conduction is progressively blocked. The excitatory phase may be brief or completely absent in the setting of rapid CNS exposure, especially with the highly lipophilic bupivacaine, which in vitro shows a particularly potent and long lasting ability to block neural tissue conduction [10].

\section{Conclusion}

We report a peculiar case of acute lidocaine and bupivacaine toxicity during orbital surgery in a woman with stable chronic systolic heart failure. As described, severe local anesthetic toxicity may occur at extremely low doses. When utilized for orbital surgery, anesthetic cardiotoxicity may mimic the oculocardiac reflex. However, several distinguishing features do exist. Recognition of these features is necessary to ensure timely delivery of antidotes to these agents.

\section{References}

1. Canavan KS, Dark A, Garrioch MA (2003) Sub-Tenon's administration of local anaesthetic: a review of the technique. Br J Anaesth 90: 787-793. [Crossref]

2. Rubin AP (1995) Complications of local anaesthesia for ophthalmic surgery. $\mathrm{Br} J$ Anaesth 75: 93-96. [Crossref]

3. Arndt GA, Stock MC (1993) Bradycardia during cold ocular irrigation under general anaesthesia: an example of the diving reflex. Can J Anaesth 40: 511-514. [Crossref]

4. Gupta N, Kumar R, Kumar S, Sehgal R, Sharma KR (2011) A prospective randomised double blind study to evaluate the effect of peribulbar block or topical application of local anaesthesia combined with general anaesthesia on intra-operative and postoperative complications during paediatric strabismus surgery. Anaesthesia 62:1110-1113. [Crossref]

5. Marriott HJ, Phillips K (1974) Profound hypotension and bradycardia after a single bolus of lidocaine. J Electrocardiol 7: 79-82. [Crossref]

6. Kim KO, Chung S, Lee K, Cho H (2011) Profound bradycardia with lidocaine during anesthesia induction in a silent sick sinus syndrome patient. J Clin Anesth 23: 227-230. [Crossref]

7. Manyari-Ortega DE, Brennan FJ (1978) Lidocaine-induced cardiac asystole. Chest 74 227-229. [Crossref]

8. Vijay SB, Mitra S, Jamil SN (2013) Refractory cardiac arrest due to inadvertent intravenous injection of $0.25 \%$ bupivacaine used for local infiltration anesthesia. Anesth Essays and Res 7:130-132. [Crossref]

9. Albright GA (1979) Cardiac arrest following regional anesthesia with etidocaine or bupivacaine. Anesthesiology 51: 285-287. [Crossref]

10. Cox B, Durieux ME, Marcus MA (2003) Toxicity of local anaesthetics. Best Pract Res Clin Anaesthesiol 17: 111-136. [Crossref]

Copyright: (C2015 Masha L. This is an open-access article distributed under the terms of the Creative Commons Attribution License, which permits unrestricted use, distribution, and reproduction in any medium, provided the original author and source are credited. 